\title{
COMMISSION 7: CELESTIAL MECHANICS AND DYNAMICAL ASTRONOMY
}

\author{
(MECANIQUE CELESTE ET ASTRONOMIE DYNAMIQUE)
}

\author{
PRESIDENT: John Hadjidemetriou \\ VICE-PRESIDENT: Andrea Milani \\ SECRETARY: Anne Lemaître \\ ORGANIZING COMMITTEE: Joseph A. Burns, Rudolf Dvorak, \\ Claude Froeschlé, Toshio Fukushima, Igor A. Gerasimov, Douglas C. Heggie, \\ Zoran Knežević, Juan C. Muzzio, Milos Š̉ilichovský, Michael Soffel \\ \& Yi-Sui Sun
}

\section{DYNAMICS OF POPULATIONS}

\subsection{Dynamics of Near Earth Objects (A. MORBIDELLI)}

The studies on the dynamics of Near Earth Objects (NEOs) performed over the 1999-2002 term can be categorized in three main groups: (i) analytic or semi-numeric studies of aspects of the dynamics of these objects; (ii) numerical integrations of specific objects of particular interest; (iii) characterizations of the statistical properties of the dynamical evolutions of ensembles of objects.

In the first group, remarkable are the works by Namouni (1999), and Namouni, Christou, \& Murray (2000) on the dynamics of NEOs trapped in coorbital motion with a planet, and those by Gronchi \& Milani (1999), (2001) and Gronchi \& Michel (2001) on the secular evolution of NEOs in the Kozai approximation. In the first two papers the authors conclude that three families of orbits are relevant for the evolution of coorbital NEOs: horseshoe orbits, passing orbits, and retrograde satellite orbits. While in the planar case these three families are disjoint, transitions between different families can occur in three dimensions, owing to the secular variations of the eccentricity and argument of pericenter. Gronchi \& Milani, using an original semi-analytical averaging method, did an exhaustive exploration of the Kozai dynamics of NEOs (the coupled evolution of the object's eccentricity and inclination, dictated by the precession or libration of the argument of perihelion) in the framework of a planetary system characterized by circular and coplanar orbits. Particularly interesting are the cases of Earth-crossing bodies which are effectively protected from close encounters with our planet by the Kozai dynamics. Averaging over a Kozai cycle, the authors computed the proper frequencies of the NEOs known at the time, and mapped the location of secular resonances in the NEO space. Gronchi \& Michel compared the results of this semi-analytic theory with those of numerical integrations and concluded that the validity of the former holds until a first strong encounter with a planet occurs. In addition to the above quoted papers, also of importance are the works by Vokrouhlický \& Milani (2000), Vokrouhlický, Milani, \& Chesley (2000) and Vokrouhlický, Chesley, \& Milani (2001), where the effects of the Yarkovsky non-gravitational force on NEO motion are modeled (see the review by $D$. Vokrouhlický). These works show that it will be possible in the close future to measure the strength of the Yarkovsky effect on specific NEOs using radar observations.

In the second group, I remind the papers by Michel et al. (1999) and by Tsiganis \& Varvoglis (2000), respectively on the evolutions of 1036 Ganymed (the largest NEO) and 719 Albert. The former paper shows that the dynamical lifetime of Ganymed is only $10 \mathrm{My}$. If the NEO population is really in steady state as typically assumed, this result implies that a $30-40 \mathrm{~km}$ body should escape from the main asteroid belt every $10 \mathrm{My}$, which poses serious 
problems to the classical collisional scenario on the origin of NEOs. The case of Albert is interesting, because this $10 \mathrm{~km}$ body was discovered in 1911 and then lost until its accidental recovery in May 2000. Its evolution is strongly chaotic, but not atypical with respect to other NEOs.

In the third group there has been a remarkable flourishing of new results, boosted by the availability of fast computers and powerful integration codes, such as "swift_rmvs3" (Levison \& Duncan 1994) and "mercury" (Chambers 1999), which have made possible to follow the evolution of thousands of bodies over their entire dynamical lifetimes. Gladman, Michel, \& Froeschlé (2000) studied a sample of 117 NEOs over a time scale of $60 \mathrm{Myr}$. They found that the median lifetime of the population is $10 \mathrm{My}$ and that more than half of the bodies end their lives in a Sun-grazing state, while about $15 \%$ are ejected from the Solar System. Foschini et al. (2000) used for initial conditions the orbits of 20 very bright bolides determined from camera networks, and concluded that the dynamics of these bodies are typical of those of the entire NEO population. Michel et al. (2000a) studied the evolution of the Mars-crosser asteroids that are initially not NEOs, and found that they become Earthcrosser on a median time-scale of $\sim 60 \mathrm{My}$ : about 2 Mars-crossers larger than $5 \mathrm{~km}$ become NEOs every million year. Michel \& Froeschlé (2000) studied the dynamical paths from the main belt to the NEO sub-region characterized by moderate eccentricity and inclination and $a \sim 1$ AU, typical of the so-called Small Earth Approachers (SEAs, Rabinowitz et al. 1993). They concluded that the SEAs can be of main belt origin, but their apparent overabundance, relative to the rest of the NEO population in the same size-range, must be an artifact imposed by the strong observational biases. Michel et al. (2000b) studied the dynamics of the Atens (NEOs with $a<1 \mathrm{AU}$ ). By computing which fraction of the Aten population temporarily achieves an IEO-like orbit (Interior to the Earth's Orbit, i.e. with aphelion distance $Q<0.983 \mathrm{AU}$ ) and the typical time spent in this configuration, they concluded that the still undetected IEO population should be about half of the Aten population. They also attempted to estimate the relative proportion between Atens and Apollos (NEOs with $\dot{q}<1 \mathrm{AU}$ and $a>1 \mathrm{AU}$ ) but, the initial conditions covering only some of the NEO sources, their result $(20 \%)$ is likely to be an overestimate. Dvorak \& Freistetter (2001) integrated the evolution of a synthetic population initially placed in the region with $0.7 \leq a \leq 1.45 \mathrm{AU}$ and $0.1 \leq e \leq 0.8$, and compiled statistics on the lifetimes and the collision frequencies with the Earth. Christou (2000) searched for NEOs that may temporarily enter in coorbital motion with the Earth and argued for a steady-state flux of objects temporarily captured in coorbital configurations with the terrestrial planets. Ipatov (1999) investigated the possible origin of NEOs from the distant Kuiper belt. He argued that $20 \%$ of the NEOs could be dormant comets of trans-Neptunian origin, but his results have never been confirmed, if not with the assumption of strong non-gravitational forces (Asher, Bailey, \& Steel 2001).

Still in the framework of this third group of dynamical works, particularly worth of mention is the ambitious project completed by Bottke et al. (2000) and (2002) on the debiased orbital and magnitude distribution of NEOs. They integrated, until their ultimate dynamical elimination, thousands of test particles initially placed in 5 intermediate sources: (a) the $\nu_{6}$ resonance, (b) the $3 / 1$ resonance, (c) the Mars-crossing population, (d) the network of diffusive resonances of the outer belt and (e) the trans-Neptunian scattered disk. From these integrations, they computed the steady state orbital distributions of the NEO subpopulations coming from each of these 5 intermediate sources. The overall NEO orbital distribution was then constructed as a linear combination of the distributions of these 5 sub-populations, thus obtaining a 4-parameters model. The NEO magnitude distribution, assumed to be orbit-independent, was constructed so its shape could be manipulated using an additional parameter. Combining the resulting NEO orbital-magnitude distribution with the observational biases associated with the Spacewatch survey, Bottke et al. obtained a model distribution which could be fit to the orbits and magnitudes of the NEOs discovered or accidentally re-discovered by Spacewatch. The best-fit parameters extracted from this technique were then used to define the de-biased NEO population. The authors concluded that there should be $\sim 960 \mathrm{NEAs}$ with semi-major axis smaller than $7.4 \mathrm{AU}$ and absolute 
magnitude $H<18$ (roughly corresponding to a diameter larger than $1 \mathrm{~km}$ ); the current level of observational completeness should therefore be $\sim 50 \% .23 \%$ of the NEAs should come from the $3 / 1$ resonance, $25 \%$ from the Mars-crossing population, $37 \%$ from the $\nu_{6}$ resonance, $8 \%$ from the outer belt and $6 \%$ from the trans-Neptunian region, although these relative contributions have moderately large error bars. The Apollo/Aten ratio should be $10 / 1$ with a relatively small uncertainty. The magnitude distribution implies a cumulative size distribution of the form $N(>D) \propto D^{-1.75}$ for objects between $200 \mathrm{~m}$ and $4 \mathrm{~km}$ in diameter, in good agreement with the size distributions of youthful cratered surfaces on the Moon, the Earth, and Mars (exponent ranging from -1.7 to -2), as well as with other works that attempted a direct de-biasing of the NEO population (Rabinowitz et al. 2000; Stuart 2001). The Bottke et al. NEO model has been extended by Morais \& Morbidelli (2002), to address the population of Earth-coorbitals; they concluded that in a steady-state about 0.6 NEOs with $H<18$ and 16.3 with $H<22$ should be in a coorbital state, at any time.

An up-to-date review on the origin and evolution of NEOs has been published by Morbidelli et al. (2002).

\subsection{Dynamics of Extrasolar Planetary Systems (R. DVORAK)}

Ten years ago the first planet moving in the pulsar $1257+12$ was discovered by Wolszczan \& Frail (1992) and since then many new planets - a typical example is the system 51 Peg (Mayor \& Queloz, 1995; Marcy \& Butler, 1996) - were detected.

Presently we have evidence for more than 100 extrasolar planets ${ }^{1}$ in almost 90 planetary systems where at least 10 are multiple planet systems. Most theoretical investigations have been devoted to the stability of these systems in connection with the determined orbital parameters and masses. Many of them are surprising for the following reasons:

- the masses are relatively large

- the massive planets move quite close to the parent star

- the orbital eccentricities seem to be rather large.

The first fact may be due to the not very sensitive observational technique, which is still unable to detect low-mass planets. The second and third facts are still unsolved problems because our present picture of the formation of planets is that the terrestrial planets and the cores of the jovian planets were formed through accretion of planetesimals, which excludes the formation of gaseous planets so close as they are observed and leads to only slightly eccentric orbits. The 'runaway growth' described e.g. by Wetherill (1990) and recently Kokubo \& Ida (1998) may be responsible for the formation of the solid cores and the subsequent capture of the surrounding solar nebula gas.

The reason for the small distances to the stars could be an inward migration, as proposed by Lin, Bodenheimer, \& Richardson (1996) for the system 51 Pegasi.

Also the large eccentricities of most of the orbits cannot be explained satisfactory. Ford, Havlickova, \& Rasio (2001) investigated the case of two equally massive Jupiter-like planets in different orbital configurations, showing that stable configurations are only possible when the orbits have eccentricities comparable to the outer Solar System planets.

- Cosmogonic results: In several 3D N-body simulations for the final stage of terrestrial planet formation Chambers (2001) using about 150 small planetary embryos (lunar to Mars size), and taking into account the perturbations from Jupiter and Saturn showed that in most cases - after some 20 to 200 million years - 3 or 4 Earth-like planets were formed.

\footnotetext{
$1_{\text {http: / oxoplanets.org }}$
} 
The orbits were less inclined and more eccentric than former studies showed (e.g. Chambers \& Wetherhill, 1998) but still not in the small range of the variations of inclinations and eccentricities of the planets in our Solar system. The stability of protoplanet systems was studied via direct numerical integrations by Chambers, Wetherill, \& Boss (1996) and by Yoshinaga, Kokubo, \& Makino (1999).

The time scales of instability $\mathrm{T}$ turned out to obey the law $\log \mathrm{T}=\mathrm{b}+\mathrm{c} \Delta a$, where all three parameters depend strongly on the initial eccentricities and inclinations of the bodies, as well as the orbital separation $\Delta a$ of the parent bodies, which also gives estimates of the formation of a system with terrestrial planets. In a simplified model of planetary accretion, which is based on the conversation of mass and momentum and the angular momentum deficit, Laskar (2000) showed how the organization of generic planetary systems could be derived from the initial mass distribution.

- Individual systems: Many studies were devoted to special systems like Gliese 876, HD 83443 and especially the $v$ Andromedae planets. In GL 876 two planets are in a 2:1 orbital resonance as first pointed out by Laughlin \& Chambers (2001), but a more detailed study by Lee \& Peale (2002) revealed that any physical process such as forced migration of the outer planet could result in capture into this resonance.

Moreover, the system stays stable up to eccentricities of 0.86 for the first planet. A rather complete study was presented by Gozdziewski, Bois, \& Maciejewski (2002), who were able to give a complete picture of stability using the MEGNO technique (Mean Exponential Growth factor of Nearby Orbits) developed by Cincotta \& Simo (2000). The star HD 83443 has two planets in the 10:1 mean motion resonance: Wu \& Goldreich (2002) found apparently aligned apsides due to the tidal and secular resonances; but also tidal distortion of the inner planet acts to reduce the ratio of the two eccentricities of the planets.

Many recent studies deal with the dynamical stability of the $v$ Andromedae planetary system, the first discovered multiple-planet extrasolar system around a main-sequence star (Butler et al. 1999).

Numerical integration techniques were used to establish the stability of systems by Laughlin \& Adams (1999), Rjvera \& Lissauer (2000), Stepinski, Malhotra, \& Black (2000), Lissauer \& Rivera (2001) and Barnes \& Quinn (2002) who found that out of 1000 numerical integrations with different parameters $5 / 6$ lead to stable systems. Particularly the 3 rd component having an eccentricity smaller than 0.3 leads always to a stable configuration. Ito \& Miyama (2001) estimated the upper limits of the two outer planets involved to be only 0.7 to 1.43 times larger than their minimum (taken for $\sin \mathrm{i}=1$ ). A global dynamical study of the $v$ Andromedae planets for a large variety of orbital parameters by Gozdziewski et al. (2001) showed that the innermost planet may cause chaotic motion for the system and that the masses for the two outermost planets may be different and still lead to stable configurations. Noble, Musielak, \& Cuntz (2002) studied the orbital stability of terrestrial planets inside the habitable zones of three stellar systems, i.e., $51 \mathrm{Peg}, 47 \mathrm{UMa}$, and HD 210277: stable orbits of terrestrial planets exist in the entire habitable zone of $51 \mathrm{Peg}$ and also in the inner part of the habitable zone of $47 \mathrm{UMa}$; no stable orbits can exist in the habitable zone of HD 210277.

- Theoretical studies: Already, before the first extrasolar planet was discovered in 1997 in double stars (Cochran et al. 1997) theoretical studies on the basis of numerical integrations for planets around one component and even both components of a double star has been carried by Dvorak (1986), Rabl \& Dvorak (1988) and Dvorak, Froeschlé, \& Froeschlé (1989). Their work was later extended to a finer grid in the mass parameter and the eccentricity of the binary by Holman \& Wiegert (1999).

Using a simplified model of the elliptic restricted problem work has been done for a great variety of parameters of the binary and the planet by Lohinger \& Dvorak (2002); 
the possible formation of planets around one component was studied by Marzari \& Scholl (2000a). The stability of periodic orbits in the 3:1 and 2:1 mean motion resonance for different mass ratios of the 2 planets was studied by Hadjidemetriou (2002) and discussed in connection with HD 82943 and Gliese 876, which have planets in the $2: 1$ resonance.

- Mass determinations: The pulsar B1257+12 with three planets, the first detected planetary system, was studied by semianalytical perturbation theory and mass estimation were given for the two planets B and C by Konacki, Maciejewski, \& Wolszczan (2000).

- Planets in clusters: An interesting fact was pointed out concerning possible planets in stellar clusters by Bonnell et al. (2001). Their results showed that planet formation could be suppressed in globular clusters while planets with distance $\leq 0.1 \mathrm{AU}$ to the parent star can be ejected from such system which will not be the case in open clusters. This was more or less confirmed by the work of Davies \& Sigurdsson (2001) who considered planetary systems in the globular cluster 47 Tucanae and found a somewhat larger survival distance of $d<0.3 \mathrm{AU}$. In an earlier work, Ford et al. (2000) investigated the pulsar B1620-26 triple system in M4 and concluded that due his results in old star clusters we may also produce extrasolar planetary systems.

We expect many new discoveries for the near future when space missions like COROT and MOST will be realised. Even more interesting results will be available when ESA's GAIA and EDDINGTON mission and NASA's TPF-Terrestrial planet finder will measure Earth sized exo-solar planets. Then the dynamical studies will be - besides the global stability of these systems - devoted to planets in the habitable zones and the search for biosignatures will be a challenge for the next decade.

\section{NEW RESULTS ABOUT STABILITY}

\subsection{Nekhoroshev Stability of Asteroids (M. GUZZO)}

The long-term stability of the asteroid main belt is not yet a completely solved problem. In particular, it is not clear if apart from regions associated to unstable motions there exists a region associated to practically stable motions, that is motions which are stable up to the solar system lifetime, which contains a significant part of the real asteroids. At present, the problem of understanding if chaotic asteroids really diffuse in phase space or not cannot be afforded with sufficiently accurate direct numerical integrations of all the asteroids over solar system lifetime. Therefore, it is necessary to use mathematical tools which predict long-term stability on the basis of the analysis of the available short and accurate numerical integrations.

Nekhoroshev (1977) theorem is a mathematical tool which allows to investigate the long-term stability of the actions of suitable quasi-integrable systems for times which increase exponentially with an inverse power of a small parameter (proportional to the norm of the perturbing function). In particular this theorem might account for the stability of the most regular non resonant initial conditions, and also for the most resonant and chaotic ones, provided that the perturbing function is suitably small. However, the original formulation of the theorem does not apply directly to asteroids perturbed by all planets.

The possibility of using the Nekhoroshev theorem to control the long-term stability of selected regions of the asteroid main belt was discussed in Morbidelli \& Guzzo (1997). In Guzzo \& Morbidelli (1997) it was proven an ad hoc version of the theorem, which takes into account all of the Hamiltonian perturbations, valid outside a limited number of mean motion resonances and low order secular resonances. Therefore, the Nekhoroshev stability of a given asteroid is reduced to understand if it belongs to the complement of these resonances, and if the hypotheses of the theorem are satisfied by the real asteroid main belt. As it is typical, it is very difficult to verify with purely analytic tools that real systems satisfy the 
hypotheses of a theorem of perturbation theory, mainly because the latter ones are proven under very restrictive conditions on the small parameters. Nevertheless numerical studies, even for systems of interest for Celestial Mechanics, have shown that it is possible to produce substantial analytic refinements of these bounds (for restricted three body problems: Celletti and Ferrara 1996; Celletti \& Chierchia 1997; for a Sun-Jupiter-Saturn secular system: Locatelli \& Giorgilli 2000; for the equilibrium point L4 in the restricted three body problem: Giorgilli et al. 1989; Celletti \& Giorgilli 1991; Giorgilli \& Skokos 1997; Skokos \& Dokoumetzidis 2001). The implementation of these methods is more difficult for systems with a high number of degrees of freedom, like the one describing the motion of main belt asteroids. Instead of refining the analytic bounds given by the theorem, researchers have investigated the problem with numerical integrations. The short term numerical computation of indicators of the dynamics (such as frequency analysis: Laskar 1990; Robutel \& Laskar 2000; fast Lyapunov indicator: Froeschlé, Lega, \& Gonczi 1997; Froeschlé, Guzzo, \& Lega 2000) on grids of initial conditions provides a picture of the geometry of resonances in the phase space. This, in turn, provides indication of the validity or not of the Nekhoroshev theorem. Among grid based calculations, Murray, Holman \& Potter (1998), Nesvorný \& Morbidelli (1998), Morbidelli \& Nesvorný (1999) put into evidence how many three body resonances overlap, typically at moderate to large eccentricity. In particular, a Nekhoroshev stability result is globally prevènted in these overlapping regions (see also Morbidelli (2002) for a detailed overview of the analytic and numerical results about the geometry of resonances in the main belt).

A new approach to the investigation of the Nekhoroshev stability has been proposed in Guzzo \& Benettin (2001), where it is proved that the Fourier spectrum of typical observables of a system in the Nekhoroshev stability regime has peculiarities, there called 'band structure of the spectrum', which can be detected with a short term numerical analysis. A similar result suitable for the application to asteroids is proved in Guzzo (2002). On the basis of these works, the Nekhoroshev stability of asteroids can be investigated with the analysis of an accurate short-term numerical integration of the selected asteroid. This analysis has been done on a sample of real asteroids of the outer main belt in Guzzo, Knežević, \& Milani (2002). Asteroids with three different dynamical regimes were analyzed: i) several members of Koronis family with regular motion; ii) chaotic asteroids from different parts of the outer main belt, with moderately large Lyapunov exponent; iii) strongly chaotic asteroids from Veritas family, in a region filled with high order mean motion resonances (Milani, Nobili, \& Knežević, 1997) and three body resonances (Nesvorný \& Morbidelli 1998) which possibly overlap. For objects of sample i) and iii) the Fourier analysis confirmed the known behavior (stability for i), instability for iii)). Among objects of sample ii), three cases with spectrum exhibiting the band structure were found. Other three cases have spectrum with no band structure, but nevertheless are not diffusing up to $100 \mathrm{Myr}$. Therefore, the method provided indication of real objects with moderately large Lyapunov exponents which are expected to be stable in view of a Nekhoroshev stability result; it also showed that chaotic objects, with orbital elements which are stable up to the integration time, are not subjected to a strong Nekhoroshev like stability result which would prevent their drift in the phase space.

The situation today is that a systematic analysis of the Nekhoroshev stability of all real asteroids has not yet been performed, but falls within the possibilities of the described existing tools of analysis.

\subsection{Trojan Asteroids: Proper Elements and Long Term Stability (C. BEAUGÉ \& F. ROIG)}

This report aims to review recent work on the dynamics and stability of the Trojan asteroids, published in refereed journals between January 2000 and June 2002. This period has been characterized by the appearance of several analytical and semi-analytical Hamiltonian models for the 1:1 mean-motion resonance, based on canonical variables derived from orbital elements. These models take advantage of the fact that co-orbital motion occurs in several 
very distinct time-scales, each of which can be associated to a different degree of freedom of the system. Through the combined use of averaging methods and the theory of Adiabatic Invariants, it is then possible to either study the short-time motion (libration of $\sigma=\lambda-\lambda_{1}$ ) considering fixed values of eccentricity and inclination $(e, I)$ or, conversely, remove the "fast" angles and focus on the long-term (i.e. secular) evolution of the system.

An example of such a study of short-term evolution can be seen in Namouni \& Murray (2000), were the authors analyzed the effects of a non-zero eccentricity and inclination on the location of the libration fixed points. Although the existence of such displacement has been known for many years, this effect is not always taken into account. The authors present simple analytical formula for their location as function of $e, I, \omega$. For certain values of these variables, they show that small amplitude librations may exist that do not encompass the classical $\mathrm{L}_{4}$ and $\mathrm{L}_{5}$ points.

Focusing on the long-term dynamics, Morais (2001) developed a linear secular theory for co-orbital motion, in the framework of the spatial-elliptic restricted three-body problem. She used a second degree Laplacian expansion of the disturbing function, and eliminated the libration terms through Adiabatic Invariance. The resulting equations yielded explicit analytical expressions for the proper $e, I$ and for the forced terms. Finally, introducing the secular motion of the perturbers, the author was able to estimate the approximate locations of linear secular resonances inside the co-orbital regions of all the planets, from Mercury to Neptune.

Beaugé \& Roig (2001) used a similar approach in their study of the long-term dynamics of the Jovian Trojan asteroids, although the Hamiltonian was represented via an asymmetric expansion of the disturbing function similar to that of Roig et al. (1998). The shortperiod libration of $\sigma$ was treated introducing a canonical transformation to 'non-librating' variables, via a procedure based on the work of Jupp (1969). The different degrees of freedom were then eliminated in succession combining Hori's averaging method with the condition of Adiabatic Invariance. The model included secular and direct perturbations of the outer planets, and allowed to compute proper elements for the real Trojans. The authors further searched for asteroidal families in both Lagrangean points, and found significant differences between the two swarms. In $\mathrm{L}_{4}$, the clusters of Menelaus and Epeios appear as the most robust candidates for real families. On the other hand, no similar clusters were detected in $\mathrm{L}_{5}$. Notwithstanding, it is difficult to state whether this difference is real or a consequence of observational biases.

A different perturbative model for co-orbital motion was presented by Nesvorný et al. (2002), using the semi-numerical determination of the averaged disturbing function developed by Moons (1994). Analyzing the spatial-circular three-body problem, they found no evidence of a libration of the argument of pericenter (the so-called Kozai resonance). Such libration, formerly identified by Kozai (1985), simply seems to be an artifact of disregarding the displacement of the fixed points of $\sigma$-libration with $e, I$. Moreover, for high eccentricities and/or inclinations, the authors found types of orbits different from the classical solutions of the planar-circular problem. These so-called "compound" orbits, previously identified by Namouni (1999), allow transitions between tadpole, horseshoe and retrograde regimes for certain values of the argument of the perihelion $\omega$. These transitions make the co-orbital orbits unstable for values of $e, I$ above a certain limit, which, in the Jovian case, could help explain the lack of real asteroids with inclinations larger than $I=40$ degrees.

Christou (2000) also discovered cases of compound orbits for the terrestrial planets. Via numerical simulations, he found that these regimes may be stable over time-scales of several $10^{4} \mathrm{yr}$, depending on the behavior of the argument of pericenter. He concluded that a steady state flux of a few NEAs in 1:1 resonance with the inner planets should be observable.

Several numerical studies on the stability of co-orbital motion have also been published in this period, with special emphasis in the case of the Jovian Trojans. Marzari \& Scholl (2000b) analyzed the effects of the $\nu_{16}$ secular resonance on large-amplitude Trojan orbits. They showed that asteroids with near-zero inclinations may be pumped up to $\sim 20-30$ 
deg in time-scales of the order of $10^{8} \mathrm{yr}$. In this manner, the authors propose to explain (at least partially) the present distribution of inclinations of real Trojans. However, it must be noted that this resonance only affects asteroids with semi-amplitude in the vicinity of 30 degrees. Thus, the effectiveness of this mechanism on a large scale is still not confirmed.

Even though several Trojans are known to be in the vicinity of secular resonances, this does not necessarily imply large scale instabilities in short time-scales. Tsiganis, Dvorak, \& Lohinger (2000) and Dvorak \& Tsiganis (2000) have integrated numerically several of these real bodies. Some are found to escape the Trojan region in time-scales of the order of 10 Myr, while other cases seem to exhibit what is usually referred to as stable chaos and may survive near their present location for very long time-scales

Using a more mathematical approach, Skokos \& Dokoumetzidis (2001) studied the spatial circular restricted three-body problem in the light of Nekhoroshev theory. This paper follows the same procedure used in Giorgilli \& Skokos (1997) for the planar case. The main result is that a non-zero inclination does not seem to appreciably reduce the radii of the effective stability region around the $L_{4}$ point, as compared to the planar problem. Thus, Arnold diffusion, which should be present in the 3D case, does not seem to introduce a significant chaoticity.

The effects of cosmogonic processes on the stability of the Jovian Trojans was the subject of two papers. Fleming \& Hamilton (2000) analyzed the stability of fictitious Jovian Trojans under the effects of Jupiter's mass accretion and secular changes in the planetary semimajor axes. Using a simple analytical model, they showed that the growth of Jupiter's mass from $\sim 10 M_{\oplus}$ to its present value causes a decrease in the libration amplitude of $40 \%$, while a radial shift of $\sim 1$ AU in Jupiter's orbit yields an amplitude increase of $4 \%$. These results solve the apparent contradictions of previous classical models by Rabe (1954) and Horedt (1984). The authors also showed that mass accretion does not affect $e$ or $I$, but radial migration may introduce changes proportional to the ratio between the initial and final semimajor axes of Jupiter.

Michtchenko, Beaugé, \& Roig (2001) also studied the stability of fictitious Jovian Trojans during primordial times, assuming that the radial migration of the orbits of Jupiter and Saturn placed them in different mutual mean-motion resonances. They showed that the passage through the 2:1, 7:3 and 5:2 planetary commensurabilities may introduce significant instabilities in the Trojan orbits. The authors concluded that the present population of Trojan asteroids is compatible with a planetary migration in which the mutual distance between Jupiter and Saturn varied at most 1.4 AU, and the passage through the $7: 3$ resonance lasted at most $4 \mathrm{Myr}$.

Finally, two papers discussed the existence of long-lived Trojan populations in the Lagrangean points of the other giant planets. Nesvorny \& Dones (2002) presented a seminumerical model for the three outermost planets. The tadpole regions of Saturn and Uranus were found to be largely unstable due to the combined effects of the secular dynamics and mean-motion resonances. Neptune, however, seems to have fairly stable Lagrangean points. The authors estimate that as much as $50 \%$ of the initial population of Neptune's Trojans may have survived to date, and encourage the observational search for these bodies. Even though the Saturn Lagrange points may be largely unstable, a small number of long-lived Saturn Trojans cannot be ruled out. In a detailed numerical study of this region, Melita \& Brunini (2001) estimated that some thousand primordial bodies larger than $5 \mathrm{~km}$ may still be present. According to the authors, this population would be of particular interest since it would be unaltered by collisional processes. 


\section{NEW RESULTS ABOUT INSTABILITY}

\subsection{Nongravitational perturbations of natural bodies - The Yarkovsky and YORP effects (D. VOKROUHLICKÝ)}

The Yarkovsky effect has been introduced as a tiny perturbation caused by the recoil force of the thermally re-radiated sunlight by the surface of a cosmic body. Though investigated since 1950s (e.g., Öpik 1951; Peterson 1976), the principal boost in recognizing its role for the orbital evolution of the minor bodies occured only in the late 1990s. The basic long-term orbital effect, namely a secular drift of the semimajor axis, is due to lag between absorption and emission of the radiation. This is a quite unique feature among other relevant perturbations on bodies in the $\simeq 0.1$ metre to $\simeq 10$ kilometre size range, and it makes the Yarkovsky effect very important for understanding a number of details in the solar system history.

During the reported years, the theory of the Yarkovsky effect has been further developed by Vokrouhlický (1999), who presented the unified solution of both diurnal and seasonal variants for spherical bodies in linear regime of the heat diffusion, Vokrouhlický \& Broz (1999), who generalized this linearized solution to the case of bodies covered with insulating surface layer (such as a regolith slab), and Vokrouhlický \& Bottke (2001), who clarified the concept of albedo as it should be used for computation of the Yarkovsky force. Nonlinear regime of the heat diffusion problem, underlying the Yarkovsky effect, was investigated by Vokrouhlický \& Farinella (1999) and Spitale \& Greenberg (2001); this last reference presents so far the most general approach, which however needs much optimization to be efficiently applied for the precise orbit computations.

The primary motivation of the Yarkovsky effect revival was to understand discrepancies between the theory of the meteorite delivery from the main asteroid belt and some meteorite data (such as the cosmic ray exposure ages). Hartmann et al. (1999) qualitatively reviewed this topic, with emphasis to the meteoritics. Bottke, Rubincam, \& Burns (2000) then integrated a limited number of test particles under the influence of both gravitational planetary perturbations and the Yarkovsky effect. This work demonstrated complicated interplay of the two phenomena, with meteorite orbits being temporarily captured in high order (or multiple) mean motion or secular resonances before being transported toward the Earth by the influence of a more powerful low order resonance or the $\nu_{6}$ secular resonance. Although the dynamical evolution of individual meteoroids thus requires careful work, the evolution of large swarms of fragments (released by catastrophic break-up or impacts on large asteroids) can be modelled statistically. The most successful effort to combine orbital dynamics (including to some extend the Yarkovsky and gravitational effects) and collisional dynamics has been the work by Vokrouhlický \& Farinella (2000). The most important result from this model is a fairly good agreement between the predicted and observed distributions of the cosmic ray exposure ages of ordinary chondrites and HED achondrites.

Farinella \& Vokrouhlický (1999) were first to point out the importance of the Yarkovsky effect for the origin of large near-Earth asteroids and semimajor-axis dispersion of the asteroid families. They noticed that the orbits of asteroids with sizes up to $\simeq 10 \mathrm{~km}$ may change the value of semimajor axis by as much as $\simeq 0.01 \mathrm{AU}$ within their estimated collisional lifetime. They hypothesized this value is enough to feed the dense network of high order resonances in the inner asteroid belt by new objects, sustaining thus on a longterm their delivery toward the Mars-crossing region (actually, the Yarkovsky effect is likely to be responsible for efficient delivery of $\mathrm{km}$-sized near-Earth asteroids directly toward the principal mean motion resonances too). Similarly, Farinella \& Vokrouhlický noticed that the estimated change of the semimajor axis was found comparable to characteristic width of typical asteroid families. As an application, Vokrouhlický et al. (2001) proposed the Yarkovsky origin of the metastable orbit of asteroid Vysheslavia, member of the Koronis family. This object, together with at least a dozen others, will fall in the $5 / 2$ mean motion resonance with Jupiter in about $10-30 \mathrm{My}$, see also Milani \& Farinella (1995). This is incompatible with $\mathrm{a} \simeq \mathrm{Gy}$ age of this family. Since the likelihood of secondary fragmentation 
origin of Vysheslavia is low, Vokrouhlický et al. argued that this asteroid might have been placed onto the unstable region of the phase space by graduate drift due to the Yarkovsky effect. The Koronis family has been then investigated in detail by Bottke et al. (2001), who found additional arguments for its long-term evolution via the Yarkovsky effect. The most important observations rely on the family sharp edges delimited by the powerful $5 / 2$ and $7 / 3$ mean motion resonances with Jupiter and a peculiar division of this family by the high order secular resonance $g-3 g_{6}+2 g_{5}$ (formerly called the Prometheus cloud).

At the present time, the existence of the Yarkovsky effect is mostly based on inferences, such as the statistical properties of large samples of objects or qualitative arguments. To conclusively prove existence of the Yarkovsky effect, and to justify its present theoretical modelling, it would be useful to directly detect its orbital perturbation on asteroids (in a consistent manner as it was done for the artificial satellites orbiting around the Earth). To that end, Vokrouhlický Milani, \& Chesley (2000) suggested that the Yarkovsky perturbation can be detected by precise orbit determination of small near-Earth asteroids that are radartracked at two or three encounters with the Earth. They simulated such observations during next close encounters of a number of targets (e.g. Golevka, Icarus, Geographos) and concluded that observations taken in the next decade should reveal the existence of the Yarkovsky effect; the most recent candidate observation occurs for Golevka in May 2003. Vokrouhlický \& Milani (2000) and Vokrouhlický, Chesley, \& Milani (2001) considered additional nongravitational effects acting on orbits of near-Earth asteroids.

Besides changing orbital parameters, Yarkovsky force can also produce torques which secularly affect the spin rate and the spin axis orientation of asteroids and meteoroids. Rubincam (2000) performed a first study of this phenomenon, proposing to name it after Yarkovsky-O'Keefe-Radzievski-Paddack (YORP for short). The YORP effect may potentially have a similarly profound influence on understanding history of small asteroids, namely distribution of their rotation rates and orientations of spin axes, as the Yarkovsky effect for their orbital dynamics. For instance, it can result in rotational fission of some asteroids (on a typical timescale of tens of $\mathrm{My}$ for $\mathrm{km}$ size object), or it can drive orientation of their spin axes preferentially to some specific directions. Moreover, by influencing the spin axis obliquity, the YORP effect intimately affects the orbital perturbation by the Yarkovsky effect. The YORP effect, and the complicated feedback it can have on the Yarkovsky orbital perturbation, certainly merits to be studied in the near future.

\subsection{Potential Impact Detection (S. CHESLEY)}

The theories and techniques relating to the detection of potential planetary impacts have matured substantially over the past triennium. These detections invariably rely upon accurately predicting the range of possible future positions of a comet or asteroid, and hence rest fundamentally upon the various methods of orbit determination, with particular emphasis placed upon the extent of possible orbital solutions, rather than on the unique best-fitting solution. It is well known that the weighting of astrometric data has a direct effect on the size and location of the region of possible orbits, and yet only recently has this aspect of orbit determination received a comprehensive treatment (Carpino, Milani, \& Chesley 2002). By considering the orbital uncertainty, Bonanno (2000) showed the significant extent to which apparently harmless objects with nominally main-belt orbits could in fact be Earth-crossing and potentially hazardous. Virtanen, Muinonen, \& Bowell (2001) have developed a new orbit determination technique, known as statistical ranging, that is suitable for objects observed on only a very few nights, when differential corrections are most likely to fail. Tholen \& Whiteley (2002) have independently implemented a similar procedure.

The basic idea of resonant returns, that a preliminary planetary encounter could deflect an object toward a collision at a subsequent encounter, was first suggested regarding asteroid $1997 \mathrm{XF}_{11}$ by Marsden (1999). Milani, Chesley, \& Valsecchi (1999) provided a semi-analytic theory for resonant returns, as well as for non-resonant returns, where the subsequent encounter is to a different planet or to the opposite mutual node. Using 1999 $\mathrm{AN}_{10}$ as a test case, they used their theory to make the first verified potential impact de- 
tection consistent with all available observations. Valsecchi et al. (2002) have developed a substantial analytic theory for resonant returns. This theory, which might fairly be called Öpik-Valsecchi theory, starts with Öpik's encounter theory (Öpik 1976) and extends it by developing a six-dimensional set of encounter elements. Analytically mapping these elements from one encounter to the next leads to a continuum of potential orbits that will be deflected toward a given subsequent encounter. In this way the "keyholes" (Chodas \& Yeomans 1999) through which subsequent impacts must pass can be identified at preliminary encounters.

Following $1999 \mathrm{AN}_{10}$, the next object for which potential impacts were detected was $1998 \mathrm{OX}_{4}$, a Potentially Hazardous Asteroid (PHA) that was unfortunately lost a year earlier. Somewhat surprisingly, the problem of lost potential impactors is actually rather tractable (Milani et al. 2000). Since an impacting orbit is known to intersect the Earth at some precise future time, its orbit is very well constrained and one can predict with high precision where this hypothetical impactor will be at intervening observable apparitions. This means that if an observer finds a lost asteroid at the predicted location then the chances of impact could be very large, and in any case the object is no longer lost. Conversely, if the asteroid is not present in the search region then one can rule out the possibility of that particular potential impact.

With the experience of $1998 \mathrm{OX}_{4}$, and the understanding that the loss of a PHA with non-negligible impact probability was objectionable, came the recognition that fielding automatic monitoring systems to warn of such cases immediately upon discovery would enable follow-up observers to recognize objects worthy of extraordinary effort. The first such system was brought online at the Univ. di Pisa in late 1999 and it detected many dozens of potential impactors, which almost invariably received suitable observational attention in order to eventually eliminate the possibility of impact. In early 2002 this first generation system was succeeded by two independently developed automatic collision monitoring systems, CLOMON2 at the University of Pisa ${ }^{2}$ and Sentry at NASA's Jet Propulsion Laboratory ${ }^{3}$. At any given time these systems list dozens of objects for which potential impacts cannot be ruled out, most of which were long lost before the systems were placed in operation.

There are several disparate approaches to encounter analysis and impact prediction. The two existing monitoring systems both rely upon sampling the range of possible elements via the so-called multiple solutions method (Milani 1999; Milani, Chesley, \& Valsecchi 2000; Chodas et al. 2001). Sitarski (1999) describes a way to obtain the best-fitting orbit allowing a collision at a particular encounter and he uses the RMS of the fit residuals to rule out the possibility of collision in some cases. Muinonen, Virtanen, \& Bowell (2001) show how such an RMS can be used to place an upper bound on the impact probability, and go on describe how to detect potential impacts within the framework of statistical ranging. An in-depth survey of impact detection methods is forthcoming (Milani et al. 2002).

Giorgini et al. (2002) analyzed a threatening close encounter between the Earth and asteroid 29075 (1950 DA) that will occur in March of the year 2880. While they did not estimate the impact probability, they did state an upper bound of $3.3 \times 10^{-3}$. In contrast to previous impact detections, where the orbit has always been very poorly constrained, this case is characterized by an extraordinarily high quality orbit that can be safely propagated for hundreds of years without significant degradation. For this reason the fidelity of the force model is a crucial issue in understanding the motion of $1950 \mathrm{DA}$. In particular, the effect of direct solar radiation pressure and Yarkovsky acceleration affect the asteroid position by $\sim 10^{7} \mathrm{~km}$ at the time of the 2880 encounter. These distances were far greater than any of the several other model refinements that were considered. The idea that radiation effects

\footnotetext{
${ }^{2}$ http://newton.dm.unipi.it/neodys

${ }^{3}$ http://neo.jpl.nasa.gov/risk
} 
can be significant in the modeling of asteroid motion was anticipated by Vokrouhlický, Chesley, \& Milani (2001).

The progressive technical advances described above have been accompanied, pari passu, by a broadening public awareness of impact risks and improved public understanding of the meaning and significance of individual cases. It is important to note that the detection of potential future impacts upon the Earth has raised serious controversy over how and when such scientific results should be published, and this remains a challenge for those conducting such work. Binzel (2000) developed the Torino Impact Hazard Scale to aid in the public communication of impact hazards. Later, Chesley et al. (2002) proposed the Palermo Technical Impact Hazard Scale, intended to aid communication among impact hazard specialists. Each of these scales have proved valuable in their own sphere as the automated impact detection systems have predicted ever increasing numbers of potential impacts.

\section{J. Hadjidemetriou \\ President of the Commission}

\section{References}

Asher, D.J., Bailey, M.E., \& Steel, D.I. 2001, in Collisional processes in the solar system, Eds Marov and Rickman, (Dordrecht : Kluwer), ASSL, 261, 121

Barnes, R., \& Quinn, T. 2002, ApJ, 550, 884

Beaugé, C., \& Roig, F. 2001, Icarus, 153, 391

Binzel, R.P. 2000, Planetary Space Science, 48, 297

Bonanno, C. 2000, A\&A, 360, 411

Bonnell, I.A., Smith, K.S., Davis, M.B., \& Horne, K.D. 2001, MNRAS, 322, 859

Bottke, W.F., Jedicke, R., Morbidelli, A., Petit, J.M., \& Gladman, B. 2000, Science, 288, 2190

Bottke, W.F., Morbidelli, A., Jedicke, R., Petit, J.M., Levison, H., Michel, P., \& Metcalfe,T.S. 2002, Icarus, 156, 399

Bottke, W.F., Rubincam, D. P., \& Burns, J.A. 2000, Icarus, 145, 301

Bottke, W.F., Vokrouhlický, D., Brož, M. Nesvorný, D., \& Morbidelli, A. 2001, Science, 294, 1693

Butler, R. P., Marcy, G.W., Fischer, D.A., Brown, T.M.; Contos, A.R.; Korzennik, S.G.; Nisenson, P., \& Noyes, R. W. 1999, ApJ, 526, 916

Carpino, M., Milani, A., \& Chesley, S.R. 2002, Icarus, submitted

Celletti, A., \& Chierchia, L. 1997, Comm. Math. Physics, 186, 413

Celletti, A., \& Ferrara, L. 1996, CM\&DA, 64, 261

Celletti, A., \& Giorgilli, A. 1991, CM\&DA, 50, 31

Chambers, J.E. 1999, MNRAS, 304, 793

Chambers, J.E. 2001, Icarus, 152, 205

Chambers, J.E., \& Wetherhill, G.W. 1998, Icarus, 136, 304

Chambers, J.E., Wetherill, G.W., \& Boss, A.P. 1996, Icarus, 119, 261

Chesley, S.R., Chodas, P.W., Milani, A., Valsecchi, G.B., \& Yeomans, D. K. 2002 Icarus, 159,423

Chodas, P.W., Chesley, S.R., Chamberlin, A. B., \& Yeomans, D. K. , Paper AAS 01-461, AAS/AIAA Conf., Quebec City, Canada.

Chodas, S.R., \& Yeomans, D.K. 1999, Paper AAS 99-462, AAS/AIAA, Conf. Girdwood, Alaska

Christou, A.A. 2000, Icarus, 144, 1 
Cochran, W.D., Hatzes, A.P., Butler, R.P., \& Marcy, G.W. 1997, ApJ, 483, 457

Cincotta, P.M., \& Simo, C. 2000, A\&AS147, 205

Davies, M.B., \& Sigurdsson, S. 2001, MNRAS324, 612

Dvorak, R. 1986, A\&A117, 621

Dvorak, R., \& Freistetter, F. 2001, Planet. Sp. Sci, 49, 803

Dvorak, R., \& Tsiganis, K. 2000, CM\&DA, 78, 125

Dvorak, R., Froeschlé, C., \& Froeschlé, Ch. 1989, A\&A, 226, 335

Farinella, P., \& Vokrouhlický, D. 1999, Science, 283, 1507

Fleming, H.J., \& Hamilton, D.P. 2000, Icarus, 148, 479

Ford, E.B., Joshi, K.J., Rasio, F.A., \& Zbarsky, B. 2000, ApJ, 528, 336

Ford, E.B., Havlickova, M., \& Rasio, F.A. 2001, Icarus, 150, 303

Foschini, L., Farinella, P., Froeschlé, Ch., Gonczi, R., Jopek, T.J., \& Michel, P. 2000, A\&A, 353, 797

Froeschlé, C., Guzzo, M., \& Lega, E. 2000, Science, 289, 2108

Froeschlé, C., Lega, E., \& Gonczi, R. 1997, CM\&DA, 67, 41

Giorgilli, A., Delshams, A., Fontich, E., Galgani, L., \& Simo, C. 1989, J. Diff. Eqs., 77, 167

Giorgilli, A., \& Skokos, Ch. 1997, A\&A, 317, 254

Giorgini, J.D., Ostro, S.J., Benner, L.A., Chodas, P.W., Chesley, S.R., Hudson, R.S., Nolan, M.C., Klemola, A.R., Standish, E.M., Jurgens, R.F., Rose, R., Chamberlin, A.B., Yeomans, D.K., \& Margot, J.L. 2002, Science, 296, 132

Gladman, B., Michel, P., \& Froeschlé, Ch. 2000, Icarus, 146, 176

Gozdziewski, K., Bois, E., Maciejewski, K., \& Kiseleva-Eggleton, L. 2001, A\&A, 378, 569

Gozdziewski, K., Bois, E., \& Maciejewski, K. 2002, MNRAS, 332, 839

Gronchi, G., \& Michel, P. 2001, Icarus, 152, 58

Gronchi, G., \& Milani, A. 1999, A\&A, 341, 928

Gronchi, G., \& Milani, A. 2001, Icarus 152, 48

Guzzo, M. 2002, CM\&DA, accepted

Guzzo, M., \& Benettin, G. 2001, Dcds B, 1, 1

Guzzo, M., \& Morbidelli, A. 1997, CM\&DA, 66, 255

Guzzo, M., Knežević, Z., \& Milani, A. 2002, CM\&DA, accepted

Hadjidemetriou, J. 2002, CM\&DA, 83, 141

Hartmann, W.K., Farinella, P., Vokrouhlický, D., Weidenschilling, S.J., Marzari, F., Davis, D.R., \& Ryan, E. 1999, Meteorit. Planet. Sci. 34, A161

Holman, M.J., \& Wiegert, P.A. 1999, AJ, 117, 621

Horedt, G.P. 1984, CM\&DA, 33, 367

Ipatov, S.I. 1999, CM\&DA, 73, 107

Ito, M., \& Miyama, S. 2001, ApJ, 552, 372

Jupp, A. 1969, AJ, 74, 35

Kokubo, E., \& Ida, S. 1998, Icarus, 131, 171

Konacki, M., Maciejewski, A.J., \& Wolszczan, A. 2000, ApJ, 544, 921

Kozai, H. 1985, CM\&DA, 36, 47

Laskar, J. 1990, Icarus, 88, 266

Laskar, J. 2000, Phys.Rev.Lett, 84, 3240

Laughlin, G., \& Adams, F.C. 1999, ApJ, 526, 881

Laughlin, G., \& Chambers, J.E. 2001, ApJ, 551, L109

Lee, M.H., \& Peale, S.J. 2002, ApJ, 567, 596

Levison, H., \& Duncan, M. 1994, Icarus, 108, 18 
Lin, D. N., Bodenheimer, P., \& Richardson, D.C. 1996, Nature, 380, 606L

Lissauer, J., \& Rivera, E. 2001, ApJ, 554, 1141

Locatelli, H., \& Giorgilli, A. 2000, CM\&DA, 78, 47

Lohinger, E., \& Dvorak, R. 2002, CM\&DA, 82, 143

Marcy, G., \& Butler, P. 1996, SPIE, 2704, 46

Marsden, B., G. 1999, J. British Interplanetary Society, 52, 195

Marzari, F., \& Scholl, H. 2000a, ApJ, 543, 328

Marzari, F., \& Scholl, H. 2000b, Icarus, 146, 232

Mayor, M., \& Queloz, D. 1995, Nature, 378, 355

Melita, M.D., \& Brunini, A. 2001, MNRAS, 322, L17

Michel, P., \& Froeschlé, Ch. 2000, CM\&DA, 79, 93

Michel, P., Gonczi, R., Farinella, P., \& Froeschlé, Ch. 1999, A\&A, 347, 711

Michel, P., Migliorini, F., Morbidelli, A., \& Zappala, V. 2000a, Icarus, 145, 332

Michel, P., Zappala, V., Cellino, A., \& Tanga, P. 2000b, Icarus, 143, 421

Michtchenko, T.A., Beaugé, C., \& Roig, F. 2001, AJ, 122, 3485

Milani, A. 1999, Icarus, 137, 269

Milani, A., \& Farinella, P. 1995, Icarus, 115, 209

Milani, A., Chesley, S. R., Chodas, P.W., \& Valsechhi, G.B. 2002, in Asteroids III, Univ. of Arizona Press

Milani, A., Chesley, S.R., \& Valsecchi, G. 1999, A\&A, 346, L65

Milani, A., Chesley, S.R., \& Valsecchi, G. 2000, Planet. Space Sci., 48, 945

Milani, A., Chesley, S.R., Valsecchi, G., \& Boattini, A. 2000, Icarus, 145, 12

Milani, A., Nobili, A.M., \& Knežević, Z. 1997, Icarus, 125, 13

Moons, M. 1994, CM\&DA, 60, 173

Morais, M.H. 2001, A\&A, 369, 677

Morais, M.H., \& Morbidelli, A. 2002, Icarus, accepted

Morbidelli, A. 2002, Modern Celestial Mechanics, Eds. Taylor \& Francis

Morbidelli, A., Bottke W.F., Froeschle Ch., \& Michel P. 2002, in Asteroid III, Eds. Bottke et al., University of Arizona Press.

Morbidelli, A., \& Guzzo, M. 1997, CM\&DA, 65, 107

Morbidelli, A., \& Nesvorný, D. 1999, Icarus, 139, 295

Muinonen, K., Virtanen, J., \& Bowell, E. 2001, CM\&DA, 81, 93

Murray, N., Holman, M., \& Potter, M. 1998, AJ, 116, 2583

Namouni, F. 1999, Icarus, 137, 293

Namouni, F., Christou, A.A., \& Murray, C.D. 2000, Phys.Rev.Lett, 83, 2506

Namouni, F., \& Murray, C.D. 2000, CM\&DA, 76, 131

Nekhoroshev, N.N. 1977, Russ. Math. Surveys, 32, 1

Nesvorný, D., \& Dones, L. 2002, Icarus, accepted

Nesvorný, D., \& Morbidelli, A. 1998, AJ, 116, 3029

Nesvorný, D., Thomas, F., Ferraz-Mello, S., \& Morbidelli, A. 2002, CM\&DA, 82, 323

Noble, M., Musielak, Z., \& Cuntz, M. 2002, ApJ, 572, 1024

Öpik, E.J. 1951, Proc. R. Irish Acad. 54A, 165

Öpik, E.J. 1976, in Interplanetary Encounters, (New York: Elsevier)

Peterson, C. 1976, Icarus, 29, 91

Rabe, E. 1954, AJ, 59, 433 
Rabinowitz, D.L., Gehrels, T., Scotti, J.V., McMillan, R.S., Perry, M.L., Wisniewski, W., Larson, S.M., Howell, E.S., \& Mueller, B.E. 1993, Nature, 363, 704

Rabinowitz, D.L., Helin, E., Lawrence, K., \& Pravdo, S. 2000, Nature, 403, 165

Rabl, G., \& Dvorak, R. 1988, A\&A, 191, 385

Rivera, E., \& Lissauer, J. 2000, ApJ, 530, 454

Robutel, Ph., \& Laskar, J. 2000, Icarus, 152, 4

Roig, F., Simula, S., Ferraz-Mello, S., \& Tsuchida, M. 1998, A\&A, 329, 339

Rubincam, D.P. 2000, Icarus, 148, 2

Sitarski, G. 1999, Acta Astron. 49, 421

Skokos, C., \& Dokoumetzidis, A. 2001, A\&A, 367, 729

Spitale, J., \& Greenberg, R. 2001, Icarus, 149, 222

Stepinski, T.F., Malhotra, R., \& Black, D.C. 2000, ApJ, 545, 1044

Stuart, J.S. 2001, Science, 294, 1691

Tholen, D.J., \& Whiteley, R.J. 2002, Icarus, submitted

Tsiganis, K., Dvorak, R., \& Pilat-Lohinger, E. 2000, A\&A, 354, 1091

Tsiganis, K., \& Varvoglis, H. 2000, A\&A, 361, 766

Valsecchi, G.B., Milani, A., Gronchi, G.F., \& Chesley, S.R. 2002, A\&A, submitted

Virtanen, J., Muinonen, K., \& Bowell, E. 2001, Icarus, 154, 412

Vokrouhlický, D. 1999, Astron. Astrophys. 344, 362

Vokrouhlický, D., \& Bottke 2001, A\&A, 371, 350

Vokrouhlický, D., \& Brož 1999, A\&A, 350, 1079

Vokrouhlický, D., \& Farinella, P. 1999, AJ, 118, 3049

Vokrouhlický, D., \& Farinella, P. 2000, Nature, 407, 606

Vokrouhlický, D., \& Milani 2000, A\&A, 362, 746

Vokrouhlický, D., Brož, M., Farinella, P., \& Knežević, Z. 2001, Icarus, 150, 78

Vokrouhlický, D., Chesley, S.R., \& Milani, A. 2001, CM\&DA, 81, 149

Vokrouhlický, D., Milani, A., \& Chesley, S.R. 2000, Icarus, 148, 118

Wetherill, G.W. 1990, LPI, 21, 1325

Wolszczan, A., \& Frail, D. 1992, Nature, 355, 132

Wu, Y., \& Goldreich, P. 2002, ApJ, 564, 1024

Yoshinaga, K., Kokubo, E., \& Makino, J. 1999, Icarus, 139, 328 